\title{
NOTAS
}

\section{Lógica del don y lógica económica}

\section{José Castro Cea}

Resumen: La nota pretende profundizar en el concepto "lógica del don" y las posibles formas de llevarlo a la práctica económica contrapuesta a la dominante lógica económica. En primer lugar se estudia el concepto de "lógica del don" en Caritas in Veritate (CiV) y su relación con la constitución conciliar Gaudium et spes (GS); en segundo lugar, se analiza la "lógica del don" desde la sociología y la filosofía recientes; por último, se presentan algunas aproximaciones desde el análisis económico que se han venido haciendo sobre comportamientos altruistas benevolentes o gratuitos.

Palabras clave: bienes relacionales, comportamientos altruistas, economía civil, "lógica del don", reciprocidad.

\section{Logic of the gift and economic logic}

Abstract: The noteaims to deepen the "logic of the gift" conceptand possible ways to economic practice opposed to the dominant economic logic. First of all examines the concept of "logic of the gift" in Caritas in Veritate (CiV) and its relationship to the Constitution reconciled Gaudium et spes (GS); Secondly, the "logic of the gift" is parsed from sociology and philosophy recent; Finally, some approaches are presented

\section{Logique de la donation et logique économique}

Résumé: La note a pour but d'approfondir le concept de la «logique du don» et les possibilités de pratique économique s'opposée à la logique économique dominante. Tout d'abord examine le concept de la «logique du don» dans Caritas in Veritate (CiV) et sa relation avec la Constitution Gaudium et spes (GS); Deuxièmement, la «logique du don» est

\footnotetext{
1 Profesor de Ética Social, Universidad Pontificia Comillas, Madrid.
} 
from economic analysis have been doing about free or benevolent altruistic behaviors.

Keywords: relational goods, altruistic behavior, civil economy, "logic of the gift", reciprocity relational goods, altruistic behavior, civil economy, "logic of the gift", reciprocity. analysée à partir de sociologie et philosophie récente; Enfin, certaines approches.

Mots clé: biens relationnels, comportement altruiste, économie civile, «la logique du don», réciprocité.

Recibido: 7 de septiembre de 2015.

\section{La lógica del don en Caritas in veritate y su relación con Gau- dium et spes}

Lo primero que cabría señalar es que el concepto de lógica del don constituye una novedad introducida por CiV dentro del acervo de la Doctrina Social Iglesia (DSI). Ciertamente este concepto pasó más bien desapercibido en los primeros momentos de la recepción de la encíclica, momentos sin duda muy marcados por las sangrantes circunstancias de crisis económica y social que se estaban, y aún se están viviendo. Y tal vez a este primer desapercibimiento también haya contribuido el hecho de que el término lógica del don aparezca tan solo tres veces en toda la encíclica, todas ellas en el capítulo tercero.

Sin embargo, según va avanzando el tiempo y se va profundizando la lectura del documento magisterial, emerge con mayor firmeza y claridad el papel central que el concepto de lógica del don juega en la propuesta doctrinal de la encíclica. Y creo que esto es así porque en el concepto de lógica del don confluye toda la reflexión expuesta en la primera parte de la encíclica, sin duda la más teológica.

Para poder comprender esta primera parte de la encíclica creo que es indispensable tener en perspectiva una de las constantes del pensamiento de Joseph Ratzinger porque aquí podemos comprobar que continúa latiendo en el fondo en el magisterio de Benedicto XVI: La relación entre fe y razón. Conociendo cómo el teólogo Joseph Ratzinger concibe la relación entre la fe y la razón, podremos comprender la propuesta de Benedicto XVI sobre la relación entre caridad y verdad, y acercarnos al mismo concepto de lógica del don. 
Sintetizando, a través de la obra de Joseph Ratzinger podemos reconocer dos momentos esenciales en su comprensión de la relación entre la razón y la fe. Un primer momento comprendido por la tesis en la que reivindica que la racionalidad de la fe, en tanto que racionalidad propia de una experiencia humana específica, genera un verdadero saber del hombre. Ordo fidei y ordo rationis no son dimensiones estancas del hombre sino las dos caras de una misma moneda que no es otra que la capacidad humana de aprehender la realidad del cosmos y del mismo hombre (RATZINGER 1985, 2000, 2006, 2011). Dicho saber teológico, lejos de yuxtaponerse al resto de los saberes humanos y sus diferentes racionalidades, se ha de articular con ellos en perfecta armonía.

En esta concepción de la relación entre la razón y la fe podemos reconocer la médula del principio de autonomía de las realidades temporales consagrado por GS (36). Así, las distintas racionalidades humanas no son ancillae (servidoras) de la racionalidad teológica, sino que "gozan de sus propias leyes y valores", y su mismo desarrollo en la búsqueda de la verdad establece su vínculo con la racionalidad de la fe. De este modo la justa autonomía de las diversas racionalidades no las sustrae, sin embargo, de una necesaria confluencia con la razón teológica, antes bien, esta relación se hace necesaria para que todas ellas avancen en el conocimiento de la verdad.

Y como segundo momento de la comprensión por parte del teólogo bávaro de esa relación entre razón y fe, estaría la tesis que propugna que, por ser verdadero saber, la racionalidad teológica aporta al conjunto de saberes del ser humano la capacidad de aprehender una dimensión trascendente que se haya presente en toda la realidad y que resulta especialmente significativa y necesaria para la propia autocomprensión del ser humano, pero también para una comprensión plena del mundo. Sin una verdadera y plena comprensión del hombre, sujeto cognoscente y arquitecto de las diferentes racionalidades, sin una antropología que albergue la dimensión trascendente y religiosa del hombre, estas mismas racionalidades carecen de las claves que dan sentido a su mismo quehacer. Esta antropología, esta comprensión del hombre es el puerto por el que se establece una relación recíproca entre la racionalidad teológica y el conjunto de saberes humanos.

En este segundo momento de la tesis de Joseph Ratzinger podemos reconocer el mismo esquema de fondo que articula GS. La Constitución pastoral del Concilio Vaticano II supone un hito en la historia de la DSI en la medida en que es un punto de inflexión metodológico, al situar en primer lugar el discurso antropológico como clave de comprensión de la DSI. GS dedica su primera parte a exponer una antropología teológica explicitando así que solo desde ésta se puede comprender 
la propuesta ético-social de la lglesia, y situando en la perspectiva antropológica la raíz del diálogo de la lglesia con el mundo.

Esta doble tesis, vemos cómo tiene su continuidad en el magisterio de Benedicto XVI. El mismo título de dicha encíclica, Caritas in veritate, y su introducción (CiV 1-4) reflejan esta pretensión fundamental de mostrar que la caridad, esencia de Dios y vivencia más específica de la experiencia cristiana, fecunda un determinado discurso, una expresión por medio de la razón, capaz de entrar en dialogo con las diferentes racionalidades humanas.

Ahora bien, va a ser en el tercer capítulo de CiV donde esta relación entre razón y fe, en su vertiente de relación entre razón y amor, se proyecte específicamente sobre la realidad social, y muy concretamente sobre la realidad económica. Esta proyección de la relación entre caridad y verdad en el mundo de las relaciones económicas cristaliza en un concepto intuitivo: lógica del don. El concepto de lógica del don constituye así el punto arquimédico sobre el que pivota toda la comprensión de la propuesta de fondo de la encíclica.

Sin embargo, a pesar de su centralidad, la encíclica no entra a desarrollar el contenido del concepto de lógica del don, por eso he calificado el concepto tal y como aparece en CiV como intuitivo; Benedicto XVI tan solo señala los rasgos básicos de esta lógica del don, que emanan de los rasgos que hemos señalado en la relación entre razón y fe, razón y amor.

El primero de estos rasgos sería que lógica del don, en la que se encarna la caridad, tiene una especificidad, una razón interna que la diferencia de la justicia. La lógica del don "trasciende toda ley de justicia" porque "supera el mérito, su norma es sobreabundar"; no se inscribe la lógica del don, por tanto, en la perspectiva del deber que funda la lógica de la justicia (CiV34). No obstante, la lógica de la justicia no le es extraña a la lógica del don, la lógica del don se articula con la norma de justicia ya que la lógica del don encuentra su "primera vía" en la justicia, aunque la trasciende (CiV6).

El segundo rasgo, que se corresponde con la segunda tesis sobre la relación entre fe y razón que hemos expuesto, sería la necesidad de la lógica del don para comprender y articular la convivencia humana y la vida en sociedad. La pretensión de la razón económica de ser autónoma, de no estar sujeta a «injerencias» de carácter moral, ha llevado al hombre a abusar de los instrumentos económicos incluso de manera destructiva (CiV35). Por tanto, Benedicto XVI planteará que el desarrollo económico, social y político necesita, si quiere ser auténticamente 
humano, dar espacio al principio de gratuidad como expresión de fraternidad (CiV34). No obstante este dar espacio no puede ser una yuxtaposición externa, no se trata de que la lógica del don entre donde no llega la justicia, se trata más bien de compartir espacio, posibilitando que la lógica del don actúe a través de la misma justicia en su sentido más amplio, articulándose con el principio de equivalencia.

En el número 36 de CiV será donde se plantee la cuestión más urgente que afrontar en esta relación ente lógica económica y lógica del don: [...] mostrar, tanto en el orden de las ideas como de los comportamientos [...] que en las relaciones mercantiles el principio de gratuidad y la lógica del don, como expresiones de fraternidad, pueden y deben tener espacio en la actividad económica ordinaria.

Este reto no hace sino concretar en el ámbito de lo económico, el planteamiento de fondo por el que la razón de la fe ilumina y fecunda realmente las distintas racionalidades humanas, en este caso, la razón económica. Por tanto se comprende que Benedicto XVI concluya este punto fundamental para la comprensión de la encíclica señalando que "esto es una exigencia del hombre en el momento actual, pero también de la razón económica misma. Una exigencia de la caridad y de la verdad al mismo tiempo".

Como se puede comprobar, CiV coloca la lógica del don como clave de arco de la articulación entre caridad y economía. Y sin embargo, siendo un concepto fundamental, la encíclica tan solo señala estos rasgos formales de la lógica del don, pero no entra en absoluto a una profundización mayor. Por tanto, si queremos afrontar el reto que lanza, se hace del todo necesario explicitar en primer lugar a qué nos referimos cuando hablamos de lógica del don, y más concretamente en tanto que significativa para la misma naturaleza de la acción económica.

Y hemos de subrayar intensamente en este momento la acotación que hemos hecho a la hora de indagar la naturaleza de la lógica del don. El amplísimo campo semántico y conceptual que el mismo término lógica del don encarna puede explorarse desde múltiples puntos de vista. En nuestro caso nos interesa exclusivamente desde el punto de vista de su significatividad en las relaciones socioeconómicas. En cierto sentido esta acotación tal vez sea más fiel a la intención de la encíclica dado el lugar en el que el término aparece dentro de ésta -el capítulo dedicado con más extensión a la vida económica-, y a que todas las ocasiones en las que aparece el término se hace en un contraste y diálogo con la lógica de la equivalencia, con la lógica del contrato, la lógica más puramente económica. 


\section{Aproximación al concepto de lógica del don}

El concepto de lógica del don, siendo una novedad en el ámbito teológico, sin embargo es un concepto con cierto recorrido en el campo de la antropología y la sociología. Aparece por primera vez en la obra del sociólogo francés Marcel MAuss en 1923, en un pequeño tratado titulado Ensayo sobre el don. Forma y razón del intercambio en las sociedades arcaicas. Esta obra toma como material de partida los múltiples datos etnográficos que documentaban prácticas de intercambio no mercantiles en diversas sociedades del pacífico occidental, realizadas mediante el intercambio de regalos, y no mediante trueque o compraventa. Esta práctica resultaba muy significativa ya que los intercambios de regalos se extendían a todos los ámbitos de la sociedad, y no meramente a ámbitos rituales, de manera que formaban un modelo de producción, distribución y circulación de bienes y servicios fuera del paradigma del mercado. Cuál es su lógica interna será la pregunta que Mauss se plantee en su ensayo. Y la estructura que dio a su respuesta se ha convertido en el paradigma de la amplia literatura y debate sobre la cuestión iniciado desde la reedición de esta obra por Lévi-Strauss en el año 1950 (LÉvI-STRAuss 1950). Esta estructura propuesta por Mauss se articula en torno a las características fundamentales que Mauss señaló en Essay.

El primer elemento para la comprensión del fenómeno del don es su carácter contradictorio, aporético, en la medida en que la entrega y aceptación de los regalos es a la vez un acto libre y obligatorio. Que un don, un regalo, es algo libre no nos es difícil de comprender, no obstante en infinidad de situaciones de interacción social la presencia de un regalo, tanto su ofrecimiento como su aceptación, es considerado un acto obligado, de modo que no ofrecerlo o no aceptarlo se considera un signo de desinterés por la relación, de desconfianza, cuando no de abierta hostilidad. Y sin embargo, la entrega y aceptación de los dones, de los regalos, no dejan de ser reconocidas por los agentes como actos libres en el sentido de voluntarios.

El segundo elemento que estructura la comprensión del fenómeno del don es entender cómo el intercambio de regalos genera un proceso de circulación de bienes, prestaciones, conocimientos, etc., fundamentado en la obligación, otra vez aporética, de devolver o corresponder a lo regalado. La dinámica de los regalos o dones es un círculo desarrollado en tres momentos: dar, recibir y corresponder. El carácter aporético no sólo se manifiesta en el dar recibir, sino también en el corresponder.

Y el tercer elemento que estructura la comprensión del fenómeno del don es la cuestión sobre la posible interpolación, o la presencia del fenómeno en las sociedades contemporáneas profundamente estructuradas en torno a la institución 
del mercado. La lógica del don como elemento de las relaciones materiales en las sociedades contemporáneas ¿es un arcaísmo o realmente se haya presente en nuestras relaciones socioeconómicas? Y si se haya presente, ¿̇qué forma adopta, de qué modo está presente y hasta qué punto es relevante en la dimensión económica de nuestra vida en sociedad?

El fenómeno del don, aunque fuera la sociología la primera disciplina en detectarlo, pronto pasó a ser una cuestión abordada desde la filosofía, mientras que su presencia en el ámbito económico ha sido muy posterior y con un cariz particular. Así pues serán la filosofía y la sociología los dos primeros campos del saber que nos proporcionen esa aproximación más precisa a la naturaleza del fenómeno del don.

La filosofía se ha aproximado a esta cuestión desde dos perspectivas. Por un lado la fenomenológica en la que, partiendo de las reflexiones de Heidegger (HEIDEGGER 2000), destacan los trabajos de Jacques Derrida (DERRIDA 1995) y Jean Luc Marion (MARION 2008) y, por otro lado, la hermenéutica, fundamentalmente con la reflexión de Paul Ricoeur (Ricoeur 1993, 2005). La lectura de estos autores nos proporciona varios elementos básicos para la comprensión del fenómeno del don.

El primer elemento básico es que el fenómeno del don no puede ser explicado en las categorías de la lógica mercantil -algo que ya habíamos visto que destacaba como rasgo de la lógica del don CiV. Para Derrida (1995), esta incompatibilidad es del todo problemática ya que, en tanto que todo don es un acto de trasmisión de algo, ese "algo" hace que la relación entre donante y donatario quede siempre marcada por la deuda y, por tanto, por el deber, la obligación, de modo que lo que empieza como don se trasmuta necesariamente en necesario intercambio, por lo que el don se hace del todo imposible. El don para Derrida es loimposible.

Marion (2008) supera esta dificultad certeramente a la vez que aporta otro elemento básico para la comprensión del don. Si al centrar el fenómeno del don en la materialidad de lo trasmitido el don se hace imposible, situemos le fenómeno del don no en la materialidad de lo donado sino en la intencionalidad de los sujetos que intervienen en la donación. El don, para liberarse de ese aniquilamiento fruto de su sumisión a la lógica de la deuda, ha de comprenderse no como una relación protagonizada por el objeto trasmitido sino como una relación intencional entre sujetos. He aquí otro rasgo esencial en el fenómeno del don, su carácter intencional.

Ricoeur (1993), al tratar el fenómeno desde otra perspectiva, la hermenéutica, enriquece la comprensión del mismo sin entrar en contradicción con lo planteado tanto por Derrida y Marion. Por un lado Ricoeur también diferencia tajantemente 
la lógica del don de la lógica de la equivalencia -propia de los intercambios mercantiles, pero también, y fundamentalmente, de la justicia. Sin embargo no las sitúa como incompatibles, sino como necesitándose una lógica a la otra. Por un lado la lógica del don necesita de la mediación de la lógica de la equivalencia si no quiere caer en la arbitrariedad del emotivismo y, por tanto, en la injusticia. Pero a su vez la lógica de la equivalencia, la de la justicia, necesita de la lógica del don si no quiere derivar en un utilitarismo, si no quiere transformar la cooperación en un mero equilibrio de intereses, cuando no en llana competencia.

Por otro lado, si el fenómeno del don, como ha matizado Marion, no se puede referir al objeto donado sino al acto de la donación presente intencionalmente en los agentes, el don para Ricoeur será entonces expresión de las relaciones personales siendo la forma propia que adquiere reconocimiento personal mutuo entre personas. El don como reconocimiento personal se inscribe en el ámbito de lo sin-precio, de lo que se sustrae a cualquier forma de valoración crematística, a cualquier pago posible. Los objetos donados, regalados, son prenda de reconocimiento, y como tales no se compran ni venden (HénAFF 2002). En este sentido, el fenómeno del don no podrá jamás entenderse como un sustituto o la forma arcaica del mercado, puesto que el don no se intercambia propiamente. Para Ricoeur no habrá un círculo don-recibir- devolver, porque devolver es en realidad realizar un nuevo primer don y no consecuencia necesaria del primer don (RICOEUR 2005).

Así pues, recapitulando, la mirada filosófica al fenómeno del don nos ha aportado algunas características fundamentales para la comprensión del mismo. A saber: la relación dialéctica entre la lógica del don y la lógica de la equivalencia, diferentes pero mutuamente necesarias; el don como un fenómeno que sólo adquiere sentido en el ámbito relacional, intersubjetivo porque es un modo específico de reconocimiento mutuo -centrarlo en su dimensión material es lo que genera la aporía; $y$, finalmente, el fenómeno del don como manifestación en las relaciones sociales de un ámbito de lo sin-precio, en el que no cabe el intercambio en cualquier forma de equivalencia que este intercambio quiera adoptar.

La mirada de la sociología sobre el fenómeno del don tiene un cariz muy diferente, ya que su intención no es tanto definir la naturaleza del fenómeno como describir su presencia en las sociedades y cómo éstas quedan configuradas de diversos modos por dicha presencia. Por este motivo la sociología no se va a preocupar por la coherencia interna de la lógica del don, por resolver la aporía del don. El principal foco de interés de la sociología se centra en ese tercer elemento del fenómeno del don tal y como Mauss lo presentó, el grado de presencia de una "economía del don" dentro de la sociedad occidental contemporánea, y por aña- 
didura en describir los mecanismos de circulación de los dones dentro de estas, segundo elemento señalado por Mauss. Cabe añadir que si la literatura sobre el don es bastante limitada en el ámbito filosófico, no lo es en absoluto en el ámbito sociológico y menos aún de la antropología cultural, donde la producción es realmente extensa. Pasemos pues a señalar cuales son las aportaciones que, en mi opinión, son relevantes para el objeto que nos ocupa.

En primer lugar, al igual que en la filosofía, la sociología y la antropología cultural van a distinguir dos ámbitos fundamentales en el modo de relacionarse las personas y las cosas: el ámbito del don y el ámbito de las mercancías (GREGORY 1982, ApPAdURA 1988, CarRIER 1991). El ámbito del don es el de los objetos vinculados íntimamente a las personas, donde el significado del objeto viene condicionado por la identidad de las personas, y por ello carece de precio, tiene un valor in-calculable. Por el contrario, el ámbito de las mercancías es el de los objetos desvinculados de toda relación personal, meros objetos totalmente trasmisibles por medio del intercambio equivalente, bien mediante un precio, bien mediante trueque. Esta distinción, ausente o casi imperceptible en las sociedades arcaicas y tradicionales, eclosiona en los albores de la edad moderna y se acelera con la industrialización.

Ahora bien, si desde el punto de vista filosófico ambos ámbitos, el del don y el de la mercancía, eran antagónicos, desde el punto de vista de la sociología y la antropología no es así ya que entre el don y la mercancía existe un continuum, una gradación que permite a los objetos pertenecer en mayor o menor medida al ámbito del don y al ámbito mercantil a un mismo tiempo.

En segundo lugar, la progresiva y creciente implantación del mercado en las relaciones de producción y distribución, una de las causas explicativas de la transformación de la sociedad feudal en la sociedad moderna, provocó por contrapartida el repliegue del ámbito del don dentro de los límites de las relaciones sociales de cercanía (familia, vecindad, pueblo...). En el ámbito regido por la lógica del mercado las personas devienen en agentes neutros, las relaciones se establecen fuera de los vínculos personales y las cosas se intercambian por el mecanismo de los precios (la oferta y la demanda) que es independiente de la relación entre los agentes (CARrier 1991, Godbout y Calllé 1992).

Este mismo proceso de acorralamiento del ámbito del don, que acontece a través del mercado, se repetirá con el surgimiento de los estados modernos democráticos y su correspondiente estatuto de ciudadanía, basada en la igualdad de derechos y deberes. El desarrollo de los aparatos burocráticos, inherente a estos estados, irá sustituyendo paulatinamente las relaciones personales (WEBER 1964). De esta 
manera buena parte de los servicios y prestaciones realizados tradicionalmente en régimen de don (cuidado de los no válidos, servicios comunales, educación, etc.) serán paulatinamente desempeñados por la "administración" a través de relaciones burocráticas impersonales realizadas por "funcionarios".

Así pues, desde un punto de vista descriptivo, en las sociedades que han pasado por el proceso de "desencantamiento" de la modernidad la lógica del don ha quedado recluida al interior de las relaciones de cercanía, a un ámbito prácticamente privado. No obstante, en este proceso hay otro campo, más allá de las relaciones de cercanía, en el que la lógica del don ha resistido y se ha podido mantener operante: el ámbito de las instituciones "benéficas", aquellas instituciones que vehiculan relaciones de gratuidad más allá de las relaciones personales directas (Godbout y CallĹ 1992).

En tercer lugar, y al hilo de este "desencantamiento", se vislumbran dos posibles modos de operar al interior de una lógica del don. Una primera modalidad que correspondería con la fórmula clásica de Mauss, y que sería una lógica circular dar-recibir-corresponder. Esta modalidad sería la que predomina en el ámbito de las relaciones de cercanía (familia, amistades, vecindad,...) y conlleva un poderoso elemento de reconocimiento y reforzamiento de los vínculos sociales primarios. Pero existiría una segunda modalidad de lógica del don, en este caso lineal, en la que el primer don no provoca una correspondencia de retorno, sino un segundo movimiento de don hacia delante (porque me ha sido dado, yo a mi vez también doy). Esta segunda modalidad de lógica del don correspondería a la de las instituciones benéficas (GODBOUt y Callé 1992, Komter y Vollebergh 1997, GOdBout 2000, KOMter 2005, KOMter 2007).

Hemos de recordar aquí que nuestro interés es la operatividad de esta lógica en las relaciones económicas en una relación dialéctica con la lógica mercantil, sin que por ello queden excluidos los muchos aspectos y matices que lo señalado hasta ahora como propio de la lógica del don pueda implicar en otros campos de conocimiento. Así pues, tras este conciso repaso a la literatura más relevante referente al fenómeno del don podemos aventurar una propuesta de los rasgos definitorios de la lógica que dota de comprensibilidad al mismo.

En primer lugar, la lógica del don se inscribe dentro del ámbito de las relaciones intersubjetivas y no de los objetos. En el fenómeno del don las realidades que se transmiten no tienen un significado inmediato en su materialidad sino en relación a los sujetos que intervienen en la donación. Frente a las relaciones en el mercado en las que la identidad de los agentes es neutra respecto a cómo circulan las 
realidades, bajo la lógica del don las cosas circulan en función de la identidad de los agentes y en función del tipo de relación que exista entre los mismos.

En segundo término, bajo la lógica del don las realidades materiales no tienen valor de cambio y, por tanto, carecen de precio. Sin embargo, que bajo el régimen del don los objetos no tengan precio no significa que no tengan valor. El valor de los objetos es primariamente el del reconocimiento simbólico del otro como sujeto de interacción social. Los objetos son símbolo de la relación que vincula a los sujetos. El valor de los objetos, siendo simbólico, en un sentido secundario va a depender de múltiples factores como pueden ser la cercanía y tipo de vínculo que exista entre las personas o las situaciones de interacción en que se realice el gesto del don, es decir, de las múltiples variaciones de claves culturales particulares en las que los sujetos se encuentren insertos.

En tercer lugar, sí bajo la lógica del don no tiene sentido la noción de valor de cambio ni precio, tampoco tiene sentido hablar de equivalencia en sentido cuantitativo, los objetos no se igualan, ni por su valor subjetivo - en el sentido de la teoría subjetiva del valor-, ni por su valor monetario. Sin embargo eso no significa que no haya un sentido de reciprocidad; ahora bien, es una reciprocidad del reconocimiento y del modo de relación entre las personas.

Finalmente, bajo la lógica del don las realidades se transmiten de dos modos, ya que siendo el reconocimiento mutuo lo que circula, este lo hace en los dos ámbitos de reconocimiento a los que ha quedado circunscrito el fenómeno del don dentro de nuestra cultura. En el ámbito de las relaciones de cercanía, aquellas en las que hay una relación de persona a persona, las realidades se desplazan en un círculo formado por los sujetos implicados en la relación, y donde la reciprocidad es directa. Pero como hemos señalado, la lógica del don opera también en un ámbito de relaciones no directas, relaciones con "otros" desconocidos, pero reconocidos a través de la mediación de las instituciones. Aquí las realidades se desplazan linealmente, no hay una reciprocidad directa, sino indirecta, donde la mediación es la institución.

\section{Lógica del don y lógica económica}

A la luz de todo lo expuesto parece que la posibilidad de implementar la lógica del don al interior de la lógica económica, respetando la autonomía de ambas, de encontrarse en algún sitio, habrá de ser en el espacio en el que los objetos 
que se transmiten no son meras mercancías, sino que de algún modo tienen una connotación relacional. El principal reto que nos encontramos entonces es superar el fuerte contraste que existe entre los elementos fundamentales que caracterizan las relaciones materiales regidas por la lógica del don con la caracterización que la teoría estándar de la economía ha hecho del comportamiento de los agentes económicos.

La teoría estándar ha tenido que desarrollar, a través de una reducción metodológica, un modelo, el agente económico, el homo oeconomicus, caracterizado por estar exclusivamente centrado en su satisfacción propia, no tanto en un egoísmo como en un no-tuismo (WICKSTEED 2003), en el sentido de la neutralidad con la que los agentes se consideran mutuamente cuando operan en el mercado; un homo oeconomicus cuyo criterio de elección es la maximización de la utilidad individual medida por una combinación del valor económico de los bienes y servicios, y un valor subjetivo individualmente considerado de los mismos, pero en todo caso medida que queda expresada un precio. Obviamente tal concepción antropológica, aunque solo sea como reducción metódica para el desarrollo de la ciencia económica, está en las antípodas conceptuales de la lógica del don.

Sin embargo, al igual que desde la filosofía y las ciencias sociales se ha producido a lo largo del siglo pasado una crítica al individualismo y al utilitarismo como paradigma de la comprensión del hombre y de la sociedad, también en el campo de la teoría económica, en el último cuarto de siglo, se ha venido produciendo una crítica al paradigma del homo oeconomicus y se han iniciado nuevas aproximaciones al comportamiento económico que posibilitan la relación con la lógica del don.

\section{Reciprocidad y bienes relacionados}

Los enfoques a los que acabamos de referirnos arrancan de la constatación, en diversos experimentos económicos, de una presencia altamente significativa de comportamientos anómalos en los agentes económicos, respecto a los predichos por la teoría estándar. En múltiples ocasiones los agentes económicos no se comportan como egoístas racionales tal y como la teoría estándar predice. Dicha constatación ha llevado a una serie de psicólogos y economistas a refutar la idea comúnmente aceptada de racionalidad del agente económico y a ajustar en los modelos el comportamiento de los agentes al realmente observado y no al presupuesto. Dichos comportamientos "anómalos" son muy variados y debidos a múltiples causas, pero de entre todos ellos los más interesantes para nosotros son 
los que su causa explicativa es la voluntad de los agentes por establecer relaciones de reconocimiento entre ellos, expresados en diferentes modos de reciprocidad y aversión a la desigualdad que se han observado en la acción económica. La interpretación ética que a posteriori se pueda hacer de estos comportamientos no es óbice para constatar que, en ellos, la acción económica no está monopolizada por la equivalencia del valor monetario sino que en el concepto de valor entran otros parámetros.

Lo primero que habría que destacar es que múltiples investigaciones han encontrado evidencias de la presencia de un comportamiento económico caracterizado por la reciprocidad, por la no competencia, por una preferencia por una relación colaborativa y no competitiva (TRIVERS 1971, GÜTH, SCHMITTBERGER y SCHWARZE 1982, Fehr, KIRCHSTEIGER y RIEDL 1993). Y si no fuera suficiente muchos de estos estudios han venido a mostrar que en muy diversas situaciones las estrategias que implican reciprocidad consiguen resultados mejores que aquellas en las que los agentes se comportan siguiendo los criterios del clásico egoísta racional (FALK y FISCHBACHER 2006).

Partiendo de estos enfoques de la economía conductual ("behavioural economics"), una propuesta destacable a la hora de indagar cómo implementar al interior de la acción económica la lógica del don es la presentada por la economía civil, cuyos más destacados representantes son los economistas italianos Luigino Bruni y Stefano Zamagni. Para la economía civil la lógica del intercambio no está solo determinada por los equivalentes, por los bienes materiales y las estrategias en función de los resultados tangibles, sino que está al mismo tiempo definida por las relaciones (BRUNI y ZAMAGNI 2004, SACCO, VANIN y ZAMAGNI, 2006, ZAMAGNI 2006, BRUNI 2008). En el intercambio no solo hay cosas, sino personas que se relacionan, y en esa relación se generan bienes relacionales (UHLANER 1989, BRUNI y Stanca 2008), en términos de A. Mclntyre "bienes inherentes a las prácticas", en este caso a la práctica económica.

Según Bruni y Stanca (2008) los bienes relacionales tienen algunas características peculiares que los constituye en una tercera categoría de bienes, ni privados ni públicos. En este sentido la dimensión relacional introduce un nuevo factor dentro de la teoría del valor. El valor del objeto no viene determinado solo por las condiciones materiales de producción e intercambio, tal como pensaba la escuela clásica, ni tampoco exclusivamente por las valoraciones subjetivas que los agentes le otorguen a dicho objeto, como postulaba la perspectiva marginalista, sino que dicho valor también está determinado, además de por el valor objetivo y el subjetivo, por las relaciones intersubjetivas que el objeto media entre los agentes, 
es decir, por el valor que los agentes le asignan a dichas relaciones. El concepto de bienes relacionales viene a recoger el carácter simbólico que tienen los bienes, por el cual las realidades materiales contienen un plus de valor que reside en su capacidad para vincular a las personas, para relacionarlas. De este modo el concepto de valor económico se enriquece con la apertura a la axiología de las relaciones intersubjetivas. Y transformar la teoría del valor supone transformar completamente el punto de vista desde donde se realiza el análisis económico.

Parece, pues, que en la propuesta de la economía civil encontramos una primera posibilidad de implementar la lógica del don en la acción económica. No obstante la economía civil tiene sus propios límites ya que básicamente solo es operativa en el campo de las relaciones más cercanas o de proximidad, con otros conocidos, en el interior de los grupos de pertenencia, en los que es posible la generación y percepción de los bienes relacionales. El concepto económico de bienes relaciones encaja bien como instrumento explicativo para ese refugio de la lógica del don en nuestras sociedades, constituido por las relaciones personales al interior de grupos de pertenencia más o menos homogéneos y que funcionaría en un sentido circular, donde la reciprocidad es la razón de la circulación de los bienes.

\section{De la interacción cara a cara a la interacción socialmente mediada}

Cuando dirigimos nuestra mirada hacia las relaciones económicas con otros anónimos, o a las macro-relaciones, la posibilidad de que esta forma de la lógica del don sea operativa adquiere una nueva forma, igualmente marcada por la reciprocidad, pero en un sentido lineal. En el mundo real los agentes económicos interactúan con una gran variedad y diversidad de agentes y manejando un abanico heterogéneo de significaciones simbólicas y culturales, o de concepciones éticas. ¿Cómo puede en un contexto de estas desarrollarse un comportamiento económico marcado por la lógica del don? Entre los enfoques críticos con la teoría estándar podemos encontrar dos que nos aportan algo de luz. Ambos se fundamentan en un concepto nacido en la sociología pero rápidamente importado por la literatura económica: el capital social.

Este concepto se fundamenta teóricamente en el enfoque conocido como racionalidad limitada, que viene a proponer que los agentes económicos en muchas ocasiones no se comportan como optimizadores racionales por diversos motivos. En estas circunstancias muchos de los comportamientos económicos no son fruto de un cálculo racional sino de un aprendizaje, bien por imitación, bien por experiencia 
propia (GIGerenzer y Selten 2002, Todd y GIGerenZer 2007). Este aprendizaje vehicula valores y normas (principalmente la confianza y la reciprocidad), y que son las que crean ciertas condiciones para el compromiso social y la participación cívica y política en el entorno y las estructuras sociales y políticas de una colectividad, es decir el capital social. Este concepto puede recoger perfectamente esa versión lineal de la lógica del don, en el sentido que el capital social se genera con la participación confiada de las personas en las instituciones siendo estas últimas las que vehiculan la reciprocidad entre las personas y los otros anónimos. Uno de los grandes méritos de la noción de capital social ha sido que ha contribuido a abrir una brecha en el "consenso de Washington", que pensaba que la democracia nacía y se desarrollaba necesariamente en la estela de los éxitos de la economía de mercado. Los trabajos sobre el capital subrayan que son la calidad de las instituciones y de la vida democrática las que condicionan el crecimiento económico (Bevort y Lallement 2006).

Así pues los comportamientos económicos inspirados en la lógica del don se pueden extender por la sociedad más allá de los grupos identitarios cercanos mediante procesos de aprendizaje y de socialización, en los que se transmita socialmente la estrategia cooperativa y reciprocadora, en el sentido lineal que señalábamos en el apartado anterior. Según este enfoque cercano a las teorías institucionalistas, se analiza el capital social como un producto de estas estructuras. Cuanto más favorecen éstas la confianza y la reciprocidad, más tendencia tienen los individuos a implicarse en la vida cívica y más capital social hay. Existen numerosísimos ejemplos de modelos culturales y sociales en los que reciprocidad social, lineal, genera un capital social cuyos efectos sobre la economía real son muy significativos (PUTNAM 1993a, Putnam 1993b, Putnam 1995, Ostrom y Ahn 2003, Bevort y Lallement 2006, Atria, Marcelo, Arriagada, Lindon y Whiteford 2015).

Por otro lado, esta posibilidad de expansión de diferentes estrategias entre los agentes económicos es el campo de estudio de la economía evolutiva. Este enfoque introduce en el análisis del comportamiento económico las variables referentes al entorno en el que se desarrolla la interacción con el fin de investigar cómo las condiciones normativas, los límites tecnológicos $u$ otro tipo de variables exógenas posibilitan el éxito evolutivo de las diferentes estrategias de los agentes, favoreciendo en ocasiones a unas en detrimento de otras. A la luz de los resultados que va aportando este enfoque (FEHR y ROCKENBACH 2003, GÜRERK, IRLENBUSCH y ROCKENBACH 2006, BRUNI 2008) podríamos concluir que tanto la supervivencia como la difusión de comportamientos altruistas en la interacción económica va a depender en gran medida de las reglas del juego y del conjunto de variables exógenas que encauzan dicha interacción. Cercano a la teoría de la movilización de los recursos, este 
enfoque se interesa por el valor más instrumental del capital social, asociando el concepto a la capacidad de las redes sociales para producir recursos como el de la información, el soporte, etc.

A la luz de todo lo expuesto parece que la posibilidad de que la lógica del don, que veíamos operativa en las relaciones más inmediatas, se extienda en la estructura de las sociedades es necesario que allí donde se transmiten los roles, los valores y las competencias sociales, así como alli donde se establecen las normas, las reglas de la interacción y las posiciones, haya lugar, o pueda operar la lógica del don en esa forma lineal que es propia de este nivel de vinculación social. Es necesario, por tanto, que el conjunto de instituciones sociales en las que todo esto se define, los ecosistemas institucionales, sean permeables a la lógica del don.

\section{Bibliografía}

Appadural, A. (1988) The Social Life of Things: Commodities in Cultural Perspective, Cambridge University Press.

Atria, R. S., Marcelo E., Arriagada I. R., Lindon J. y Whiteford S. (2015) Capital social y reducción de la pobreza en América Latina y el Caribe: en busca de un nuevo paradigma, Santiago de Chile, CEPAL.

BeVort, A. y LALLEMENT M. (2006) Le capital social: performance, équité et réciprocité, Paris, La Découverte.

BRUNI, L. (2008), Reciprocity, altruism and the civil society: in praise of heterogeneity, Routledge.

BRUNI, L. y StANCA L. (2008) "Watching alone: Relational goods, television and happiness". Journal of Economic Behavior \& Organization, 65(3): 506-528.

BRUNI, L. y ZAMAGNI S. (2004) Economia civile. Efficienza, equità, felicità pubblica, Bolonia, II Mulino.

Carrier, J. (1991) Gifts, Commodities, and Social Relations: A Maussian View of Exchange, Sociological Forum, JSTOR.

DerRIDA, J. (1995) Dar (el) tiempo: I. La moneda falsa, Barcelona, Paidós Ibérica. 
FALK, A. y FISCHBACHeR U. (2006) "A theory of reciprocity": Games and Economic Behavior 54 (2), 293-315.

Fehr, E., KirChstelger G. y Riedl A. (1993) "Does fairness prevent market clearing? An experimental investigation": The Quarterly Journal of Economics 437-459.

FeHR, E. y ROCKENBACH B. (2003) "Detrimental effects of sanctions on human altruism": Nature, 422 (6928) 137-140.

Gigerenzer, G. y Selten R. (2002) Bounded rationality: The adaptive toolbox, MIT Press.

GodBout, J. (2000) Le don, la dette et l'identité: homo donator versus homo oeconomicus. Paris, La Découverte/MAUSS.

Godbout, J. y Callé A. (1992) L'esprit du don, Paris, La Découverte.

Gregory, C. A. (1982) Gifts and commodities, Londres, Academic Press.

GÜRERK, Ö., IRLENBUSCH B. y ROCKENBACH B. (2006) "The competitive advantage of sanctioning institutions". Science, 312 (5770), 108-111.

Güth, W., Schmittberger R. y Schwarze B. (1982) "An experimental analysis of ultimatum bargaining". Journal of Economic Behavior \& Organization, 3(4), 367-388.

Heidegger, M. (2000) Ser y tiempo, Madrid, Trotta.

HÉnAfF, M. (2002) Le prix de la vérité: le don, I'argent, la philosophie. Paris, Seuil.

Komter, A. (2007) "Gifts and Social Relations The Mechanisms of Reciprocity". International Sociology, 22 (1), 93-107.

Komter, A. E. (2005) Social Solidarity and the Gift, Cambridge University Press.

Komter, A. E. Vollebergh W. (1997) "Gift giving and the emotional significance of family and friends". Journal of Marriage and the Family 747-757.

Lévi-Strauss, C. (1950) "Introduction à l'œuvre de Marcel Mauss". en Marcel Mauss, Sociologie et anthropologie, G. GURVITCH. Paris, Presses universitaires de France, «Quadrige»; ix-lii. 
MARION, J.L. (2008) Siendo dado. Ensayo para una fenomenología de la donación. Madrid, Síntesis.

Mauss, M. (1923) "Essai sur le don", Sociologie et anthropologie, 143-279.

OSTROM, E. у AнN T.K. (2003) Foundations of social capital, Edward Elgar Cheltenham.

Putnam, R. (1993a) "The prosperous community: social capital and public life". The american prospect 13 (Spring), 4. Consultable en linea: http://www.prospect. org/ print/vol/13. Acceso 7-IV-2003.

PUtNAM, R. D. (1993b) "The prosperous community: social capital and public life": The american prospect (13).

PutNam, R. D. (1995) "Bowling alone: America's declining social capital": Journal of democracy $6(1), 65-78$.

RAtZinger, J. (1985) Teoría de los principios teológicos: materiales para una teología fundamental, Barcelona, Herder.

RATZINGER, J. (2000) "Fe, verdad y cultura" en Congregación para la Doctrina de la Fe. Reflexiones a propósito de la Encíclica "Fides et ratio", 160-161.

RatZinger, J. (2011) Fe y ciencia: un diálogo necesario, Santander, Sal Terrae.

RATZINGER, J. (2006) Dialéctica de la secularización: sobre la razón y la religión, Madrid, Encuentro.

Ricoeur, P. (1993) Amor y justicia, Madrid, Caparrós.

Ricoeur, P. (2005) Caminos del reconocimiento: tres estudios, Madrid, Trotta.

SACCO, P. L., VANIN P. y ZAMAGNI S. (2006) "The economics of human relationships", en Handbook of the Economics of Giving, Altruism and Reciprocity, 1: 695-730.

Todd, P. M. y Gigerenzer, G. (2007) “Environments That Make Us Smart Ecological Rationality" en Current Directions in Psychological Science 16 (3): 167-171.

TRIVERS, R. L. (1971) "The evolution of reciprocal altruism". Quarterly review of biology: $35-57$. 
UHLANER, C. J. (1989) "'Relational goods" and participation: Incorporating sociability into a theory of rational action": Public choice 62 (3), 253-285.

Weber, M. (1964) Economía y sociedad: esbozo de sociología comprensiva, Fondo de Cultura Económica.

Wicksteed, P. H. (2003) The Common Sense of Political Economy: And, Selected Papers and Reviews on Economic Theory. London, Routledge.

ZAMAGNI, S. (2006) L'economia come se la persona contasse: verso una teoria economica relazionale. Working Paper presented at Aiccon meeting, Bolonia. 